\title{
Active extravasation of contrast within the hemorrhage (spot sign): a multidetector computed tomography finding that predicts growth and a worse prognosis in non-traumatic intracerebral hemorrhage
}

\author{
Extravasamento ativo de contraste no interior da hemorragia (spot sign): \\ um achado de TCMD que prediz crescimento e pior prognóstico nas \\ hemorragias intraparenquimatosas cerebrais não traumáticas \\ Marcos Rosa Júnior', Antônio José da Rocha', Nelson Saade², \\ Antônio Carlos Martins Maia Júnior ${ }^{1}$, Rubens José Gagliardi³
}

\begin{abstract}
Intracerebral hemorrhage $(\mathrm{ICH})$ causes high rates of disability and neurological sequelae. Objective: To evaluate spot signs as predictors of expansion and worse prognosis in non-traumatic ICH in a Brazilian cohort. Method: We used multidetector computed tomography angiography to study 65 consecutive patients ( 40 men, 61.5\%), with ages varying from 33 to 89 years (median age 55 years). Clinical and imaging findings were correlated with the findings based on the initial imaging. Results: Of the individuals who presented a spot sign, $73.7 \%$ died (in-hospital mortality), whereas in the absence of a spot sign the mortality rate was $43.0 \%$. Although expansion of ICH was detected in $75 \%$ of the patients with a spot sign, expansion was observed in only $9.0 \%$ of the patients who did not present a spot sign. Conclusions: The spot sign strongly predicted expansion in non-traumatic $\mathrm{ICH}$ and an increased risk of in-hospital mortality.
\end{abstract}

Keywords: spot sign, multidetector computed tomography angiography, hemorrhagic stroke, brain hemorrhage.

\section{RESUMO}

A hemorragia intraparenquimatosa cerebral (HIC) apresenta altas taxas de incapacidade e sequela neurológica. Objetivo: Avaliar a presença de spot sign como preditor de expansão e pior prognóstico no follow-up de HIC não-traumática em brasileiros. Método: Usamos a ângio-tomografia para estudar prospectivamente 65 pacientes consecutivos ( 40 homens 61,5\%), com idades variando de 33 a 89 anos (mediana 55 anos). Evolução clínica e achados de imagem foram correlacionados com a interpretação dos achados do exame inicial. Resultados: 73,7\% dos indivíduos com spot sign no estudo tomográfico inicial evoluíram para óbito e, na sua ausência, a taxa de mortalidade foi 43,0\%. Enquanto a expansão da HIC foi detectada em $75 \%$ dos pacientes com spot sign, ela foi notada em $9 \%$ daqueles sem este sinal. Conclusão: 0 spot sign mostrou-se forte preditor de expansão da HIC não traumática e representa maior risco de morte hospitalar nesta coorte de pacientes.

Palavras-chave: spot sign, ângio-TC, acidente vascular cerebral hemorrágico, hemorragia cerebral.

Intracerebral hemorrhage (ICH) accounts for approximately $10-15 \%$ of all strokes ${ }^{1}$ and has a poor prognosis, with a 30 -day mortality rate of approximately $50 \%$ and high rates of disability and neurological sequelae ${ }^{2}$. Computed tomography
(CT) is often the first imaging test performed on patients with suspected ischemic or hemorrhagic stroke ${ }^{1}$. Fast acquisition, wide availability, a relatively low cost, and a high sensitivity for the detection of acute hemorrhage have justified the 
increase in multidetector computed tomography (MDCT) use during the last decade.

Recent studies have confirmed that expanding hematoma occurs in approximately $70 \%$ of patients undergoing CT scan within the first 3 hours of stroke, suggesting that certain findings on MDCT angiography are likely predictors of growth hemorrhage ${ }^{3,4}$. The most important predictor of mortality and neurological disability is the initial volume of the $\mathrm{ICH}$; however, hematoma expansion leads to a conservative prognosis. The presence and characteristics of an active extravasation of contrast within the $\mathrm{ICH}$ (spot sign) was initially detected on angiography as a predictor of hemorrhage expansion ${ }^{5}$. This imaging sign has also been reproduced on MDCT angiography as a robust predictor of expansion and as an indicator of worse prognosis and mortality ${ }^{3,4}$. MDCT angiography has been proven to be a rapid and reproducible alternative to catheter angiography that reduces the risks to the enrolled patients and offers significant benefits to the decision-making process ${ }^{6-9}$.

A higher incidence of hemorrhagic stroke has been reported in the Latin American population ${ }^{10-13}$. Inherited ethnic factors attributed to miscegenation have been implicated in addition to several environmental characteristics, such as diet, often pre-existing long-term untreated arterial hypertension, and delayed access of Brazilians to emergency rooms, all of which presumably contribute to the worsening health conditions in our population. These features might influence the clinical and/or imaging patterns.

To the best of our knowledge, this is the first MDCT study of primary ICH to examine a large sample of Latin American patients. It remains necessary to reproduce the applicability of MDCT angiography and to evaluate the effects of hemostatic drugs on this population.

Our primary aims are to study the spot sign characteristic that can be observed on MDCT scans, and to assess the relationship of this finding with mortality and expansion in a cohort of patients with primary ICH. The relevance of the current study derives from the potential reproducibility of this procedure within routine evaluations of this common condition, which frequently demands intensive care and is responsible for variable neurological sequelae in economically active persons.

\section{METHODS}

\section{Patients}

The present study is part of a larger study of the use of MDCT techniques to evaluate hyperacute stroke; consequently, the protocols have been separately reviewed and approved by the Institutional Review Board and the local ethics committee.
Patients of any age or gender who presented with brain parenchyma hemorrhage $\geq 2.0 \mathrm{~cm}$ in any axis as demonstrated by routine non-contrast CT (NCCT) from August 2011 to September 2012 were considered eligible for submission to the protocol. MDCT scanning with comparable imaging parameters, including computed tomography angiography (CTA), was performed within the first 3 days after the ictus. Informed consent was obtained from all subjects (signed personally or by their guardians).

Primary ICH was diagnosed when systemic hypertension, which was defined as blood pressure $\geq 140 \times 90 \mathrm{mmHg}$, was the only identified etiology. We excluded patients whose ICH had occurred more than 3 days previously, those with contraindications to the intravenous iodine contrast agent, those who refused to participate in the study, and those whose examinations included images that were either of inadequate quality or contained technical artifacts.

The clinical data and laboratory results were reviewed to make a systemic arterial hypertension diagnosis. Glycemia (Gl) and blood pressure (BP) were considered according to the level. These parameters were studied separately, and their influence on both $\mathrm{ICH}$ expansion and in-hospital mortality was analyzed. Any other etiology, solely or superimposed on systemic hypertension that could justify ICH, was considered to be additional exclusion criteria. All patients were monitored in the intensive care unit or emergency room and treated according to the Brazilian guidelines for the management of intracerebral hemorrhage ${ }^{14}$.

All of the imaging examinations were conducted using a minimal dose of both ionizing radiation and intravenous iodinated contrast in a 64-slice CT scanner (Brilliance CT 64 Channel, Philips Medical, Eindhoven, The Netherlands), adding CTA to the previously requested NCCT. The CTA examination was performed using $120 \mathrm{kV}$ and 185 milliamperes (mA), a 450-mm field of view (FOV), and a pitch of 0.673 . The arterial phase was performed after a time delay of 20 seconds by injecting iodine contrast at $4-6 \mathrm{ml} / \mathrm{s}$ in a dual-head power injector (Medrad, Warrendale, USA) with an 18-G i.v. access, generally located in a peripheral vein, at a total dose of $1.5 \mathrm{ml} / \mathrm{kg}$. The venous phase was obtained using identical parameters 60 seconds after the beginning of contrast administration. The control CT study was performed using similar parameters, but without contrast (NCCT), according to clinical judgment.

\section{Imaging analysis}

The imaging interpretations included analysis of the NCCT and CTA source images (CTA-SI), as well as maximum intensity projection (MIP) and three-dimensional (3D) postprocessed views. All data were post-processed using commercially available software on a workstation (Extended Brilliance Workspace v3.5.0.2250, Philips Medical Systems Nederland B.V., PC Best, The Netherlands). 
All of the studies were evaluated in consensus by two radiologists (AJR and MRJr) experienced in the diagnosis of $\mathrm{ICH}$ and in the interpretation of CTA. A spot sign was considered to be present or absent on CTA-SI according to the following previously defined criteria proposed by Delgado Almandoz and Romero ${ }^{4}$ : one or more focus of contrast pooling within a hemorrhage (of any size and morphology), discontinuous from normal or abnormal vasculature adjacent to the hemorrhage, and attenuation $\geq 120 \mathrm{UH}$.

The hematoma volumes were calculated at the first NCCT scanning and at imaging follow-up, estimating the three major axes of the hematoma and multiplying the result by 0.52 . Extra-axial hemorrhage was not considered in the volume calculations.

To comparatively categorize our cohort of subjects, we considered the number of extravasation foci within the $\mathrm{ICH}$ as well as the largest dimension of focus and attenuation in Hounsfield Units (HU) to build a spot sign score (SSS), as previously proposed ${ }^{4,15}$ (Table 1).

Hematoma expansion was defined at the follow-up NCCT by an absolute growth greater than $6 \mathrm{ml}$ or a relative growth of more than $33 \%$ from the initial $\mathrm{NCCT}^{3}$. A poor outcome was defined as substantial hematoma expansion at follow-up CT, death, or the need for surgery.

\section{Statistical analysis}

Univariate analysis using either Fisher's exact test or Student's t test was used to determine the relationship between the presence of the spot sign with the expansion of ICH and hospital mortality. We also used a multivariate logistic regression model to identify the independent predictors of the expansion of the hemorrhage and hospital mortality. The results were considered to be statistically significant when $\mathrm{p}<0.05$.

\section{RESULTS}

According to the inclusion criteria, 155 subjects were consecutively studied during the defined period. A total of 90

\begin{tabular}{ll} 
Table 1. The spot sign score (SSS), as proposed by & \\
Delgado-Almandoz et al. ${ }^{15}$. & \\
\hline Spot sign characteristic & Points \\
\hline Number of spot signs & 1 \\
$\quad 1-2$ & 2 \\
$\geq 3$ & \\
Maximum axial dimension & 0 \\
$\quad 1-4$ mm & 1 \\
$\geq 5$ mm & \\
Maximum attenuation & 0 \\
$120-179$ HU & 1 \\
$\geq 180 \mathrm{HU}$ &
\end{tabular}

SSS: spot sign score; HU: Hounsfield Unit. patients $(90 / 155,58.1 \%)$ were subsequently excluded: 25 individuals $(25 / 155,16.1 \%)$ were excluded due to ICH that was attributed to trauma, 46 additional subjects $(46 / 155,29.7 \%)$ were ultimately diagnosed with secondary $\mathrm{ICH}, 15$ others $(15 / 155,9.7 \%)$ were excluded because peripheral venous access was not possible, two subjects $(2 / 155,1.3 \%)$ had been evaluated more than 72 hours after ictus, and two subjects $(2 / 155,1.3 \%)$ were excluded due to inappropriate technical conditions or the presence of artifacts that hampered the analysis.

We enrolled 65 subjects with primary ICH to the current study, with no statistically significant differences with respect to gender or age $(\mathrm{p}=0.111)$. Demographic data, BP and Gl levels, ICH volume, and the elapsed time between the stroke and the initial CTA were all measured (Table 2). The spot sign on CTA-SI was observed in 19 subjects $(19 / 65$, 29.2\%). The median intracerebral blood volume was $20.7 \mathrm{ml}$, and the average volume of blood was estimated as $49.4 \mathrm{ml}$ (range 1-245 ml). The average volume was $60.3 \mathrm{ml}$ in the spot sign-positive group (range 2.6-245 ml) and $44.9 \mathrm{ml}$ in the spot sign-negative group (range $1-159 \mathrm{ml}$ ), with no significant difference between these groups.

According to Gl levels, patients were divided into two distinct groups ( $\mathrm{Gl} \leq 140 \mathrm{mg} / \mathrm{dl}$ and $\mathrm{Gl} \geq 141 \mathrm{mg} / \mathrm{dl})$. According to their BP levels they were also divided into two others groups (BP=140-179×90-109 $\mathrm{mmHg}$ and $\mathrm{BP} \geq 180 \times 110 \mathrm{mmHg})$. No statistical relationship was demonstrated between any of these groups and $\mathrm{ICH}$ expansion or in-hospital mortality ( $p>0.05)$.

All of the spot signs $(19 / 19,100 \%)$ were detected during the venous phase of MDCT angiography in our series of patients (Figure 1). Conversely, the sign was detected in 15 subjects during the arterial phase (15/19, 79\%).

The frequency, sensitivity, and specificity of the spot sign in our data were compared with previously reported results (Table 3$)^{4}$. Brain hemorrhage was categorized according to its SSS (Table 4). When analyzed together, the SSS groups (score 1, 2, 3, and 4) exhibited an expansion of hemorrhage in $75 \%(6 / 8)$ of the cases compared with $9.0 \%(2 / 23)$ of the cases without a spot sign, and this difference was significant $(\mathrm{p}=0.002)$. Taken together, the SSS groups showed a mortality rate of $73.7 \%$ (14/19) versus $43.0 \%$ (20/46) among the patients who did not present this imaging finding ( $\mathrm{p}=0.029)$.

When categorizing patients according to the SSS, we found six patients with score 1 (6/19), 10 with score $2(10 / 19)$, one with score $3(1 / 19)$, and two patients with score $4(2 / 19)$. Hemorrhage expansion was observed in $9.0 \%(2 / 23)$ of the patients without a spot sign, in $80 \%(4 / 5)$ of those with score 1 , and in $67 \%(2 / 3)$ of those with score 2 . All of the patients with scores of 3 and 4 died after the initial CT and did not undergo a CT control.

A spot sign was documented in our series of patients only on the first day, even after the first 3 hours of ictus, with an 
Table 2. The demographic data, clinical and blood test from our series of patients.

\begin{tabular}{|c|c|c|c|c|c|c|c|c|c|}
\hline Patient & Gender & Age & Ictus (h) & Volume (ml) & Spot sign & SS artery* & SS venous* & SSS & Race $^{\dagger}, \mathrm{Gl}^{\ddagger}, \mathrm{BP}^{\S}$ \\
\hline 1 & $\mathrm{~F}$ & 82 & 13 & 113.0 & $\mathrm{~N}$ & & & 0 & Wh, 100, 190/100 \\
\hline 2 & $\mathrm{~F}$ & 37 & 24 & 44.0 & N & & & 0 & Wh, 106, 150/100 \\
\hline 3 & $\mathrm{~F}$ & 87 & 8 & 50.0 & Y & + & + & 2 & $\mathrm{Bl}, 101,240 / 140$ \\
\hline 4 & $\mathrm{~F}$ & 60 & 7 & 17.4 & N & & & 0 & Wh, $94,140 / 110$ \\
\hline 5 & $\mathrm{~F}$ & 33 & 6 & 88.0 & N & & & 0 & Wh, 95, 220/110 \\
\hline 6 & $\mathrm{~F}$ & 72 & 1 & 21.7 & N & & & 0 & Wh, 123, 210/160 \\
\hline 7 & $\mathrm{~F}$ & 55 & 9 & 56.0 & N & & & 0 & $\mathrm{Bl}, 130,140 / 90$ \\
\hline 8 & $\mathrm{~F}$ & 48 & 12 & 20.0 & N & & & 0 & Wh, 116, 150/90 \\
\hline 9 & $\mathrm{~F}$ & 47 & 11 & 17.0 & Y & + & + & 2 & $\mathrm{Bl}, 105,180 / 110$ \\
\hline 10 & $\mathrm{~F}$ & 37 & 2 & 96.0 & Y & + & + & 2 & Wh, 88, 200/120 \\
\hline 11 & F & 44 & 6 & 6.3 & Y & - & + & 1 & Wh, 96, 200/140 \\
\hline 12 & F & 45 & 4 & 32.0 & Y & + & + & 2 & Wh, $100,140 / 90$ \\
\hline 13 & $\mathrm{~F}$ & 63 & 1 & 4.3 & Y & - & + & 1 & Wh, $463,160 / 110$ \\
\hline 14 & M & 76 & 3 & 220.0 & Y & + & + & 2 & Wh, 236, 240/120 \\
\hline 15 & $\mathrm{~F}$ & 80 & 5 & 14.0 & Y & + & + & 4 & Wh, 278, 220/110 \\
\hline 16 & F & 77 & 24 & 17.8 & N & & & 0 & $\mathrm{Mx}, 133,160 / 110$ \\
\hline 17 & $\mathrm{~F}$ & 44 & 1 & 2.9 & Y & + & + & 1 & Wh, 270, 230/120 \\
\hline 18 & $\mathrm{~F}$ & 44 & 3 & 3.5 & N & & & 0 & Wh, 227, 150/100 \\
\hline 19 & $\mathrm{~F}$ & 78 & 3 & 2.4 & N & & & 0 & Wh, $96,180 / 100$ \\
\hline 20 & $\mathrm{~F}$ & 43 & 4 & 1.3 & N & & & 0 & Wh, 83, 240/170 \\
\hline 21 & $\mathrm{~F}$ & 72 & 12 & 82.0 & N & & & 0 & Wh, 155, 190/110 \\
\hline 22 & $\mathrm{~F}$ & 56 & 48 & 56.0 & N & & & 0 & $M x, 156,180 / 120$ \\
\hline 23 & $\mathrm{~F}$ & 55 & 14 & 31.0 & N & & & 0 & Wh, $215,140 / 90$ \\
\hline 24 & $\mathrm{~F}$ & 54 & 18 & 13.2 & N & & & 0 & Wh, $107,240 / 140$ \\
\hline 25 & $\mathrm{~F}$ & 72 & 5 & 11.9 & N & & & 0 & Wh, 126, 160/100 \\
\hline 26 & $\mathrm{~F}$ & 51 & 24 & 15.0 & N & & & 0 & Wh, 124, 230/130 \\
\hline 27 & M & 53 & 48 & 18.0 & N & & & 0 & Wh, 120, 250/160 \\
\hline 28 & M & 59 & 9 & 13.0 & N & & & 0 & Wh, $61,240 / 140$ \\
\hline 29 & M & 38 & 6 & 140.0 & N & & & 0 & Wh, 126, 180/130 \\
\hline 30 & M & 41 & 72 & 10.5 & N & & & 0 & $M x, 175,140 / 90$ \\
\hline 31 & M & 59 & 5 & 15.9 & N & & & 0 & Wh, $100,220 / 160$ \\
\hline 32 & M & 67 & 3 & 99.0 & N & & & 0 & Wh, $209,200 / 130$ \\
\hline 33 & M & 49 & 2 & 5.6 & N & & & 0 & Wh, 135, 280/140 \\
\hline 34 & M & 67 & 3 & 68.8 & Y & + & + & 1 & Wh, 109, 230/120 \\
\hline 35 & M & 74 & 48 & 33.0 & N & & & 0 & $M x, 112,160 / 110$ \\
\hline 36 & M & 49 & 10 & 6.7 & N & & & 0 & Wh, $105,170 / 110$ \\
\hline 37 & M & 45 & 6 & 126.0 & N & & & 0 & $M x, 113,150 / 100$ \\
\hline 38 & M & 54 & 18 & 17.7 & N & & & 0 & Wh, 207, 180/100 \\
\hline 39 & M & 48 & 6 & 58.0 & N & & & 0 & Wh, $121,160 / 110$ \\
\hline 40 & M & 46 & 4 & 20.7 & N & & & 0 & As, $155,140 / 90$ \\
\hline 41 & M & 49 & 8 & 30.5 & N & & & 0 & Wh, 136, 200/110 \\
\hline 42 & M & 46 & 24 & 58.0 & N & & & 0 & Wh, 95, 170/90 \\
\hline 43 & M & 55 & 24 & 41.6 & Y & + & + & 2 & Wh, $161,150 / 110$ \\
\hline 44 & M & 66 & 3 & 115.0 & N & & & 0 & Wh, $114,140 / 90$ \\
\hline 45 & M & 64 & 3 & 16.5 & Y & + & + & 2 & Wh, 160, 140/90 \\
\hline 46 & M & 52 & 6 & 159.0 & $N$ & & & 0 & Wh, $118,160 / 110$ \\
\hline 47 & M & 77 & 7 & 81.0 & N & & & 0 & Wh, $110,160 / 100$ \\
\hline 48 & M & 81 & 6 & 58.0 & $N$ & & & 0 & Wh, $260,190 / 100$ \\
\hline 49 & M & 45 & 2 & 14.1 & N & & & 0 & Wh, 319, 160/110 \\
\hline 50 & M & 51 & 24 & 24.0 & N & & & 0 & Wh, $130,170 / 120$ \\
\hline 51 & M & 79 & 3 & 245.0 & Y & + & + & 4 & Wh, $145,160 / 100$ \\
\hline 52 & M & 68 & 4 & 7.5 & $N$ & & & 0 & Wh, $214,160 / 90$ \\
\hline 53 & M & 61 & 6 & 2.5 & Y & + & + & 2 & Wh, $148,140 / 90$ \\
\hline 54 & M & 57 & 8 & 186.0 & Y & + & + & 3 & Wh, $210,180 / 90$ \\
\hline 55 & M & 47 & 10 & 10.8 & N & & & 0 & $\mathrm{Bl}, 220,160 / 110$ \\
\hline 56 & M & 89 & 12 & 118.0 & N & & & 0 & Wh, $133,150 / 100$ \\
\hline 57 & M & 81 & 11 & 123.0 & N & & & 0 & Wh, 137, 150/90 \\
\hline 58 & M & 57 & 11 & 87.0 & N & & & 0 & Wh, 150, 220/120 \\
\hline 59 & M & 60 & 3 & 107.0 & Y & + & + & 2 & $M x, 120,220 / 140$ \\
\hline 60 & M & 60 & 2 & 10.9 & Y & + & + & 2 & Wh, $123,220 / 120$ \\
\hline 61 & M & 52 & 11 & 19.0 & N & & & 0 & Wh, 150, 160/100 \\
\hline 62 & M & 45 & 1 & 9.0 & Y & - & + & 1 & $M x, 148,280 / 160$ \\
\hline 63 & M & 46 & 6 & 1.0 & N & & & 0 & $M x, 100,220 / 100$ \\
\hline 64 & M & 39 & 3 & 17.0 & Y & - & + & 1 & Wh, 112, 190/90 \\
\hline 65 & M & 59 & 7 & 2.3 & N & & & 0 & Wh, 97, 140/90 \\
\hline
\end{tabular}

F: female; M: male; N: no; Y:yes; SSS: spot sign score.

*Spot sign detected on arterial (SS artery) or venous (SS venous) phases of computed tomography angiography; ${ }^{\dagger}$ Races were considered according to selfreported skin color: White (Wh), Brown skin or Mixed (Mx), Black (Bl) or Asian (As); ₹Gl: Glycemia (mg/dl); \$BP: blood pressure (mmHg). 
average of 5.1 hours and a median of 3 hours (range 1-24 hours). The median overall time from the ictus to CTA scanning was 6 hours in our series, and the mean was 10.9 hours (range 1-72 hours).

Multivariate logistic regression was used to analyze certain additional features, including the ICH location and volume, the presence of a spot sign, the shape and attenuation of the hemorrhage, and the duration of ictus. The incidence of mortality was primarily influenced by the ICH volume $(\mathrm{p}=0.001)$ and the presence of a spot sign $(p=0.02)$, whereas the expansion of hemorrhage was only influenced by the spot $\operatorname{sign}(\mathrm{p}=0.001)$.

\section{DISCUSSION}

The active extravasation of contrast within a hemorrhage has been described in catheter angiography for nearly 30 years ${ }^{5}$. Similar findings have also been described in magnetic resonance imaging with the use of gadolinium ${ }^{17}$ and in CTA-SI ${ }^{2,45}$.

Recent guidelines have confirmed that rapid neuroimaging is strongly recommended to confirm the hemorrhagic nature of the stroke, while CTA may be considered useful to evaluate for underlying secondary $\mathrm{ICH}$ determined by structural lesions, including vascular malformations and tumors (Class IIa; Level of evidence B) and also to help identify

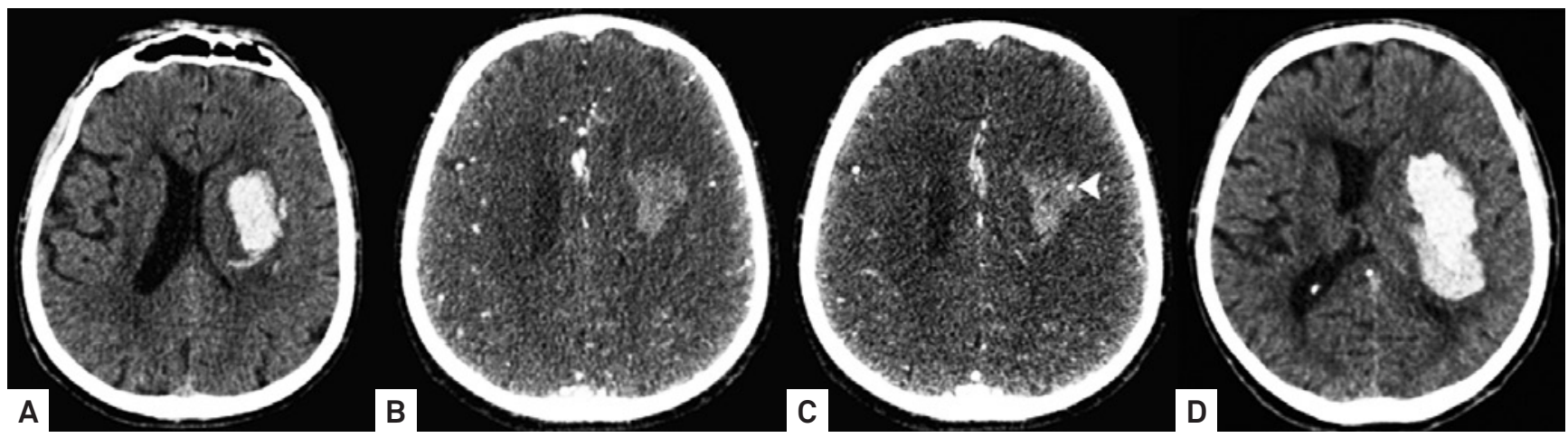

Figure 1. A 67-year-old male presented with right hemiparesis and a decreased level of consciousness. Initial non-contrast computed tomography (NCCT) after 2 hours of ictus (A) demonstrating the larger extension of an intracerebral hemorrhage (ICH) $(6.5 \times 4.0 \times 4.3 \mathrm{~cm}$, volume $=60 \mathrm{ml})$. The arterial phase of the computed tomography angiography source images (CTA-SI) (B) did not reveal a spot sign. The venous phase of the CTA-SI (C) revealed an unequivocal spot sign (arrow). NCCT for the imaging follow-up (D) showing $\mathrm{ICH}$ expansion after 24 hours of ictus $(7.6 \times 4.0 \times 6.0 \mathrm{~cm}$, volume=94 $\mathrm{ml})$.

Table 3. Frequency and accuracy of the spot sign for the prediction of mortality and hematoma expansion in several studies.

\begin{tabular}{|c|c|c|c|c|}
\hline \multirow{2}{*}{ Studies } & \multicolumn{4}{|c|}{ Sensitivity/specificity } \\
\hline & $\mathrm{N}$ & Spot Sign frequency (\%) & Hematoma expansion & Mortality \\
\hline Becker et al. ${ }^{22}$ & 113 & 46.0 & $\mathrm{n} / \mathrm{a}$ & $77 / 73$ \\
\hline Wada et al. ${ }^{2}$ & 39 & 33.3 & $91 / 89$ & $43 / 69$ \\
\hline Goldstein et al. ${ }^{23}$ & 104 & 55.8 & $92 / 50$ & $73 / 50$ \\
\hline Kim et al. ${ }^{24}$ & 56 & 26.8 & $\mathrm{n} / \mathrm{a}$ & $50 / 83$ \\
\hline Ederies et al. ${ }^{25}$ & 61 & 42.6 & $94 / 79$ & $\mathrm{n} / \mathrm{a}$ \\
\hline Delgado Almandoz et al..$^{15}$ & 367 & 19.3 & $88 / 93$ & $\mathrm{n} / \mathrm{a}$ \\
\hline Delgado Almandoz et al. ${ }^{26}$ & 573 & 23.2 & $\mathrm{n} / \mathrm{a}$ & $41 / 85$ \\
\hline Hallevi et al. ${ }^{27}$ & 27 & 48.1 & $100 / 100$ & $\mathrm{n} / \mathrm{a}$ \\
\hline Rosa Jr. et al. & 65 & 29.2 & $67 / 91$ & $41 / 85$ \\
\hline
\end{tabular}

n/a: not applicable.

Table 4. Spot sign score and its relationship with mortality, expansion of hemorrhage and poor outcome.

\begin{tabular}{|c|c|c|c|c|c|c|c|c|}
\hline SSS & $\mathrm{N}$ & N2 & Expansion & Mortality & Poor outcome & Expansion \% & Mortality \% & Poor outcome \% \\
\hline 0 & 46 & 23 & 2 & 20 & 30 & 9 & 43.5 & 65.2 \\
\hline 1 & 6 & 5 & 4 & 4 & 4 & 80 & 66.7 & 66.7 \\
\hline 2 & 10 & 3 & 2 & 7 & 9 & 67 & 70 & 90 \\
\hline 3 & 1 & 0 & 0 & 1 & 1 & - & 100 & 100 \\
\hline 4 & 2 & 0 & 0 & 2 & 2 & - & 100 & 100 \\
\hline $\operatorname{AUC}(95 \% \mathrm{Cl})$ & & & & & & $0.79(0.59-0.9)$ & $0.63(0.49-0.76)$ & $0.56(0.41-0.70)$ \\
\hline Pvalue & & & & & & 0.012 & 0.006 & 0.04 \\
\hline
\end{tabular}

SSS: spot sign score; N: total number of patients; N2: number of patients with a control CT; AUC: area under curve; Cl: confidence interval. 
patients at risk for hematoma expansion (Class IIb; level of evidence $B)^{16}$. Our results agree with previous reports confirming that the presence of spot sign on CTA-SI is an accurate and reliable predictor of non-traumatic hemorrhage expansion, mortality, and poorer neurological outcomes ${ }^{2,4}$.

The current report confirmed that contrast extravasation not only is an early event in CTA-SI analysis, as the spot sign occurred after the first 3 hours of ictus in our cohort of patients, but was also documented 24 hours after the ictus. The detection of a spot sign in our series of patients was less frequent than in certain previous reported series ${ }^{2,22,23,25,27}$, most likely due to their shorter delay before arriving at the hospital. Spot sign detection is higher if the CTA is obtained within the first 3 hours after stroke ${ }^{2,27}$, but it has also been documented after this period and can be detected during both phases of MDCT angiography (arterial and venous) ${ }^{3,4}$. The reported frequency of these delayed spot signs, which are detected during the venous phase of MDCT angiography, ranges from $8 \%$ to $23 \%$ of all the spot signs identified in various series ${ }^{4}$. The venous phase of CTA was also the most important for the detection of the spot sign in our series, allowing for its detection in $21 \%$ of the patients.

Appropriated institutional imaging protocols for MDCT, including CTA, must be carefully defined in order to avoid unnecessary radiation exposure and intravenous contrast administration. The use of this technique is supported by the current literature ${ }^{4,6-9}$. Delgado-Almandoz et al., ${ }^{4,15}$ defined CTA-SI parameters appropriate for the diagnosis of the spot sign within ICH. To predict a variable risk for the various types of spot signs, these authors have proposed a score that considers the number of foci of leakage inside of the hemorrhage, and the size of greater focus and its attenuation. To the best of our knowledge, this is the first study that uses the same CTA approach to both detect and categorize spot signs in a Brazilian cohort of patients.

Our data are consistent with previously published results, confirming similar results in the detection of spot sign within $\mathrm{ICH}$ and reinforcing the applicability of CTA to clarify the risk of a worse prognosis, including in-hospital mortality ${ }^{3,4,16}$. The demographic data among our patients were similar to those in previous studies ${ }^{1,4}$. Despite the higher mortality among those patients with hyperglycemia, our results did not confirm statistical significance. Probably the small number of patients in the defined groups has limited the analysis. Different levels of high BP were also not correlated with the worse prognosis in this series. Conversely, international guidelines have defined that normoglycemia is recommended (Class I, Level of evidence C), while BP should be monitored according to previously defined parameters (Class IIb; Level of evidence C $)^{16}$. Despite that, optimal management of high BP and hyperglycemia in $\mathrm{ICH}$ remains to be clarified ${ }^{14,16}$.

The current literature suggests applying careful interpretation to recognize results that can mimic spot sign on CTASI. Some of these results may be due to vascular causes, such as aneurysms and arteriovenous malformations that have continuity with adjacent vessels, facilitating recognition ${ }^{18}$. There are also non-vascular causes that can mimic spot signs on CTA-SI, such as heterogeneous bleeding and calcification foci. All of these findings should be scrutinized on both NCCT and CTA to avoid the misdiagnosis of spot signs.

Our report has certain limitations, including the delayed arrival of the patients to our center and the smaller sample of patients relative to previous series ${ }^{4,15,23,26}$. Although the majority of our patients self-reported themselves as Caucasians, the incidence of miscegenation in our population is well known ${ }^{10-13}$. Certain ethnic variations, including miscegenation and concurrent uncontrolled systemic arterial hypertension, may contribute to more severe ICH outcomes among Latin Americans ${ }^{10-13}$. Further controlled studies using hemostatic drugs in Latin American subjects remain necessary to examine the expansion of hemorrhage with variable clinical outcome improvements, as previously reported among populations in developed countries ${ }^{19-21}$.

The authors encourage the use of this standardized MDCT protocol, including both arterial and venous phases, in an imaging work-up for ICH among Brazilian patients who arrive at the hospital during the first 24 hours after ictus. An appropriate approach and specific treatment might support the adoption of public policies in treating Brazilian patients with $\mathrm{ICH}$, ensuring earlier and more efficient treatment.

In conclusion, the incidence of a spot sign on CTA-SI was positively associated with hematoma expansion, a poor prognosis, and mortality in our Brazilian cohort of patients with primary ICH. Our results reinforce the hypothesis that CTASI is useful to detect the spot sign during the first 24 hours after primary ICH, particularly when considering venous phase CTA 3 hours after ictus. These findings also support the conclusions that a higher score (SSS) significantly increases the chance of death and that even the presence of a spot sign is an independent strong predictor of hematoma expansion.

\section{References}

1. Fischbein NJ, Wijman CA. Nontraumatic intracranial hemorrhage. Neuroimag Clin N Am 2010;20:469-492.

2. Wada R, Aviv RI, Fox AJ, et al. CT angiography "spot sign" predicts hematoma expansion in acute intracerebral hemorrhage. Stroke 2007;38:1257-1262.
3. Demchuk AM, Dowlatshahi D, Rodriguez-Luna D, et al. Prediction of haematoma growth and outcome in patients with intracerebral haemorrhage using the CT-angiography spot sign (PREDICT): a prospective observational study. Lancet Neurol 2012;11:307-314.

4. Delgado Almandoz JE, Romero JM. Advanced CT imaging in 
the evaluation of hemorrhagic stroke. Neuroimag Clin N Am 2011;21:197-213

5. Kowada M, Yamaguchi K, Matsuoka S, Ito Z. Extravasation of angiographic contrast material in hypertensive intracerebral hemorrhage. J Neurosurg 1972;36:471-473.

6. Krol AL, Dzialowski I, Roy J, et al. Incidence of radiocontrast nephropathy in patients undergoing acute stroke computed tomography angiography. Stroke 2007;38:2364-1366.

7. Dittrich R, Akdeniz S, Kloska SP, et al. Low rate of contrast-induced nephropathy after CT perfusion and CT angiography in acute stroke patients. J Neurol 2007;254:1491-1497.

8. Hopyan JJ, Gladstone DJ, Mallia G, et al. Renal safety of CT angiography and perfusion imaging in the emergency evaluation of acute stroke. Am J Neuroradiol 2008;29:1826-1830.

9. OleinikA, Romero JM, Schwab K, et al. CT angiography for intracerebral hemorrhage does not increase risk of acute nephropathy. Stroke 2009;40:2393-2397.

10. Lessa I, Silva MR. Cerebrovascular diseases as multiple cause of death in Salvador: magnitude and space differences of mortality omitted in official statistics. Arq Neuropsiquiatr 1993;51:319-324.

11. Saposnik G, Del Brutto OH. For the Iberoamerican Society of Cerebrovascular Diseases. Stroke in South America: A systematic review of incidence, prevalence, and stroke subtypes. Stroke 2003;34:2103-2108.

12. Lessa I, Bastos CA. Epidemiology of cerebrovascular accidents in the city of Salvador, Bahia, Brazil. Bull Pan Am Health Organ 1983;17:292-303.

13. Minelli C, Fen LF, Minelli DPC. Stroke incidence, prognosis, 30day, and 1- year case fatality rates in Matão, Brazil. Stroke 2007;38:2906-2911

14. Pontes-Neto OM, Oliveira-Filho J, Valiente R, et al. Brazilian guidelines for the management of intracerebral hemorrhage. Arq Neuropsiquiatr 2009;67:940-950

15. Delgado Almandoz JE, Yoo AJ, Stone MJ, et al. Systematic characterization of the computed tomography angiography spot sign in primary intracerebral hemorrhage identifies patients at highest risk for hematoma expansion: the spot sign score. Stroke 2009;40:2994-3000.
16. Morgenstern LB, Hemphill JC, Anderson C, et al. Guidelines for the Management of Spontaneous Intracerebral Hemorrhage: A Guideline for Healthcare Professionals From the American Heart Association/ American Stroke Association. Stroke 2010;41:2108-2129.

17. Murai Y, Ikeda Y, Teramoto A, Tsuji Y. Magnetic resonance imagingdocumented extravasation as an indicator of acute hypertensive intracerebral hemorrhage. J Neurosurg 1998;88:650-655.

18. Gazzola S, Aviv RI, Gladstone DJ, et al. Vascular and nonvascular mimics of the CT angiography "spot sign" in patients with secondary intracerebral hemorrhage. Stroke 2008;39:1177-1183.

19. Davis SM, Broderick J, Hennerici M, et al. Hematoma growth is a determinant of mortality and poor outcome after intracerebral hemorrhage. Neurology 2006;66:1175-1181.

20. Mayer SA, Brun NC, Begtrup K, et al. Efficacy and safety of recombinant activated factor $\mathrm{VII}$ for acute intracerebral hemorrhage. N Engl J Med 2008;358:2127-2137.

21. Anderson CS, Huang Y, Wang JG, et al. Intensive blood pressure reduction in acute cerebral haemorrhage trial (INTERACT): a randomised pilot trial. Lancet Neurol 2008;7:391-399.

22. Becker KJ, Baxter AB, Bybee HM, et al. Extravasation of radiographic contrast is an independent predictor of death in primary intracerebral hemorrhage. Stroke 1999;30:2025-32.

23. Goldstein JN, Fazen LE, Snider R, et al. Contrast extravasation on CT angiography predicts hematoma expansion in intracerebral hemorrhage. Neurology 2007;68:889-894.

24. Kim J, Smith A, Hemphill JC III, et al. Contrast extravasation on CT predicts mortality in primary intracerebral hemorrhage. Am $J$ Neuroradiol 2008;29:520-525.

25. Ederies A, Demchuk A, Chia T, et al. Postcontrast CT extravasation is associated with hematoma expansion in CTA spot negative patients. Stroke 2009;40:1672-1676.

26. Delgado Almandoz JE, Yoo AJ, Stone MJ, et al. The spot sign score in primary intracerebral hemorrhage identifies patients at highest risk of in-hospital mortality and poor outcome among survivors. Stroke 2010;41:54-60

27. Hallevi H, Abraham AT, Barreto AD, et al. The spot sign in intracerebral hemorrhage: the importance of looking for contrast extravasation. Cerebrovasc Dis 2010;29:217-220. 